\title{
Particularities and limitations of kinesiotherapy in genetic disorders and their medical recovery - Russell-Silver Syndrome: a case report
}

\author{
Adina Sorina Mitrică, Avram Carmen, Ada Bargau Petrache, \\ Adrian Cantemir, Vlad Teodor Iacob
}

\begin{abstract}
Adina Sorina Mitrică - Senior Kinesiotherapist, County Clinical Hospital "Saint Apostol Andrei”, Constanța

Avram Carmen - Senior M.D. in Physical Medicine and Balneology, Clinical Rehabilitation Hospital "Băile Felix", Children`s ward 1 Mai

Ada Bargau Petrache - Junior Pediatric Cardiologist, County Clinical Hospital "Gavril Curteanu", Oradea

Adrian Cantemir - M.D., PhD, Assistant Professor, "Grigore T. Popa" University of Medicine and Pharmacy Iaşi, Romania

Vlad Teodor Iacob - M.D., PhD student, “Socola” Institute of Psychiatry, Iași, Romania
\end{abstract}

\begin{abstract}
While some authors still separate the Russell-Silver syndrome as two independent disorders, it is presently considered as one whole entity. It is a rare syndrome, with over 400 reported cases (1). The first reported cases were in children with the syndrome's characteristic facial features, low birth weight, body asymmetry, and poor growth after birth (2), growth failure being the primary abnormality (3). Patients typically present with intrauterine growth retardation, difficulty feeding, failure to thrive, or postnatal growth failure $(4,5)$. The clinical features are more evident in infants or young children (6).
\end{abstract}

Methods. We present a 19-month-old girl, admitted to our hospital for functional rehabilitation due to mildly severe neuromotor retardation, associated with severe malnutrition, low body weight, congenital heart malformation and gastroesophageal reflux. We evaluated the child and her motion of joints and strength of muscles using, among other tests, the Gross Motor Function Measure (GMFM) scale. She underwent a kinesiotherapy program in order to enhance and rise her motor skills according to her chronological age. 
r.m\%\%\% Results. For one year, rehabilitation treatment improved muscle tone, motor skills, posture and spine alignment. Also, a specific kinesiotherapy program at home resulted in a systematic improvement in growth and development in this case.

Conclusions. Genetic anomalies are always a challenge for therapists. An early intervention program, in this particular case, is highly beneficial.

\section{KEY WORDS:}

\section{Kinesiotherapy, rehabilitation, Russell-Silver Syndrome.}

\section{INTRODUCTION}

Russell-Silver Syndrome (RSS) is a rare congenital imprinting disorder with a wide array of clinical presentations, but also with many characteristic features such as:

- Low birth weight and short stature, which frequently lead to difficulties regarding diagnosis and treatment

- Hypotonia, which is frequently related to neurological disorders, is more often present in newborns until they reach adolescence than in teenagers or adults. The increased frequency of hypotonia is correlated with the survival of children born prematurely with consecutive neurological impairments.

While RSS was first described separately by the two authors, due to similarities, it is now considered the same entity.

One of RSS's main features is growth retardation with a prenatal onset and a slow developmental regression, to which are added some anomalies that give it a characteristic facial appearance, easily recognizable.

\section{CASE REPORT}

\section{Reasons for admission}

The patient, aged 1 year and 7 months, was admitted for functional recovery treatment due to growth delay with short stature and poor weight gain, and moderate delay in psychomotor skills.

\section{Medical history}

Family history: healthy father, while the mother was diagnosed with multinodular goiter and hyperthyroidism.

The patient is the first-born, with a premature birth by cesarean section and dysgravidy, at 36 weeks, with a weight of 1900 grams, length of $45 \mathrm{~cm}$, Apgar score 7/8, resuscitated at birth using a bag valve mask and tactile stimulation.

Early postnatal, she has a functional respiratory disorder, neonatal hypoglycemia, E. coli infection in the amniotic fluid, and was treated with antibiotics and mixed feeding.

At 2-months-old, she was diagnosed with dysphagia, hypoxic-ischemic encephalopathy, neuromotor retardation, developmental coordination disorder, craniofacial dysmorphism, moderate protein-calorie malnutrition and treated with Pyritinol at the Children's Clinical Hospital in Oradea.

At 3-months-old, in the Pediatric Clinic II in Cluj-Napoca, she is presumed to have esophageal stenosis, for which a nasogastric tube for feeding was inserted.

At 4-months-old, while admitted for interstitial pneumonia in Oradea, she was diagnosed with an atrial septal defect, type ostium secundum, right to left shunt; pulmonary hypertension and paroxystic 
supraventricular tachycardia, and megaesophagus.

At 5-months-old, while admitted to the Pediatric Clinic III in Cluj-Napoca for vomiting and growth failure (Ponderal Index $=0,66)$, a metabolic genetic disorder is suspected.

At 6-months-old, she is fed by gavage, her weight is stagnant, her caloric intake is limited due to refusing food, all of which with a lack of hypoproteinemia excludes malabsorption syndrome.

From the age of 9 months, she slowly gained weight and at 1-year-old, she was genetically investigated due to severe hypotrophy.

At the age of 1 year and 2 months, an MRI showed adenohypophyseal hypoplasia and demyelination of the periventricular white matter (secondary lesions due to hypoxia).

At the age of 1 year and 3 months, she was investigated at the Universita di Roma and underwent genetic testing, which led to a presumed diagnosis of Russell-Silver Syndrome

\section{Initial assessment}

- Hypotrophy due to growth delay, with a height of $67 \mathrm{~cm}$, weight of $5,2 \mathrm{~kg}$ and head circumference of $47 \mathrm{~cm}$ (ideal for her age being a height of $81,5 \mathrm{~cm}$ and weight of $11 \mathrm{~kg}$ )

- Characteristic triangular facies, prominent supraorbital ridge, fontanelle $2 / 3 \mathrm{~cm}$, pigmented skin.

- Axial hypotonia, accentuated dorsolumbar kyphosis in the sitting position.

- Hypotonia in all four limbs, symmetrical muscular hypotrophy, dynamic knee valgus.
- From the supine position, supported from the chest by the physiotherapist, she does not raise her head.

- She does not get into a sitting position; She does not get into orthostatism without assistance.

- Walking is only possible with bilateral support; walks with a wide base of support, asymmetric, with stability and balance disorders.

\section{Kinetic objectives}

- Obtaining orthostatism.

- Improved postural control.

- Enhancing sensorimotor and functional deficits.

- Improvement of muscle tone and kinetic balance by learning balance control strategies (ankle, knee, hip strategy) and by learning controlled falls.

- Toning the muscle groups involved in breathing.

- Increasing physical training while monitoring blood glucose levels.

- Correcting her dynamic and static body posture (reverting her hyperlordosis).

\section{Results at her latest evaluation}

- Height of $80 \mathrm{~cm}$ and weight of $7000 \mathrm{~g}$.

- Normal spinal curvatures

- The upper limbs show normal muscle development and the patient can use them to achieve activities of daily living (ADL).

- The lower limbs show a muscular toning that allows the child to walk independently over long distances, to walk on rough terrain, to walk up and down a ramp, going up and down the stairs and running. 
- From the supine position, she can flex her head and neck while supporting her elbows.

- She can independently obtain orthostatism.

\section{CONCLUSIONS}

Early detection and treatment of children with RSS decisively influence recovery and future integration possibilities.Motivation for movement is very important throughout treatment. Teamwork that involves all the specialists who work with the child: recovery doctor, physiotherapist, occupational therapist and the patient's family can determine favourable developments.

During physical therapy programs, the child should practise various postures to comprehend all the different sensations that come with them.

The effectiveness and rigour of personal hygiene programs, nutrition (one hour before and after physical therapy, it is recommended to not consume anything) must be observed, and in this case, kinesiotherapy is contraindicated if it is not supported by a hypercaloric diet.

Special attention must be paid to the development of the body scheme.

In the recovery process, there is no standardized program, it varies from individual to individual, which is why we consider it important to take into account all the neuro-psycho-motor and physiological elements of each individual and the whole range of means kinesiotherapy has to offer. Physical therapy will focus on achieving psychomotor skills, especially static function, dynamic function, global coordination, manual dexterity, trunk flexibility, speed of arm movement, lower limb strength.

Achieving a correct alignment of the spine has been the key to achieving the other goals successfully and will soon allow the child's integration into a kindergarten that addresses normal children.

\section{ACKNOWLEDGEMENTS AND DISCLOSURES}

The authors state that there are no declared conflicts of interest regarding this paper.

\section{REFERENCES}

1. Tubbs RS, Wellons JC, Oakes WJ. Russell Silver syndrome and tethered spinal cord. Child's Nervous System. 2004 Jul;20(7):473-5.

2. Price SM, Stanhope R, Garrett C, Preece MA, Trembath RC. The spectrum of Silver-Russell syndrome: a clinical and molecular genetic study and new diagnostic criteria. Journal of medical genetics. 1999 Nov 1;36(11):837-42.

3. Şıklar Z, Berberoğlu M. Syndromic disorders with short stature. Journal of clinical research in pediatric endocrinology. 2014 Mar;6(1):1.

4. Prasad NR, Reddy PA, Karthik TS, Chakravarthy M, Ahmed F. A rare case of Silver-Russell syndrome associated with growth hormone deficiency and urogenital abnormalities. Indian journal of endocrinology and metabolism. 2012 Dec;16(Suppl 2):S307.

5. Yamaguchi Jr KT, Salem JB, Myung KS, Romero Jr AN, Skaggs DL. Spinal deformity in Russell-Silver syndrome. Spine deformity. 2015 Jan 1;3(1):95-7. 
Bulletin of Integrative Psychiatry O New Series OSeptember 2021 OYear XXVII ONo. 3(90)/137

(n) 6. Ranke MB, Price DA, Reiter EO, editors. Growth hormone therapy in pediatrics: 20 years of KIGS. Karger Medical and Scientific Publishers; 2007.

\section{Correspondence:}

Adina Sorina Mitrică,

Senior Kinesiotherapist County, Clinical Hospital "Saint Apostol Andrei”, Tomis Blvd 145, Constanţa, Romania, adinacozac2@gmail.com

Submission: 12 aug 2021

Acceptance: 11 sep 2021 